\title{
Experimental Validation and Constitutive Relationship of Steel Material under Elevated Temperature and High Strain Rate
}

\author{
Shi Yan ${ }^{1,2, a}$, Hang $W^{1, b}$ and Baoxin $\mathrm{Qi}^{1,3, c}$ \\ ${ }^{1}$ School of Civil Engineering, Shenyang Jianzhu University, 110168, China; \\ ${ }^{2}$ State Key Laboratory of Explosion Science and Technology, Beijing University of Technology, \\ 100081, China; \\ ${ }^{3}$ Jiangsu Key Laboratory of Environmental Impact and Structural Safety in Engineering, China \\ University of Mining and Technology, 221008, China. \\ a syan1962@163.com, b356412107@qq.com, ' qibaoxin2005@163.com
}

Keywords: Q345 steel material; SHPB experiment; Johnson-Cook model; Constitutive model; Elevated temperatures; High strain rate.

Abstract. Based on the temperature rise caused by adiabatic process and the viscosity of the metal under the impact of steel, the Johnson-Cook constitutive relation of structural steel in civil engineering was modified. Applying the Split-Hopkinson Pressure Bar (SHPB) testing technique,

the impact experiment under elevated temperatures $\left(25,350,450\right.$ and $\left.750^{\circ} \mathrm{C}\right)$ and high stain rates (2300 and $3200 \mathrm{~s}^{-1}$ ) of Q345 steel was performed. Johnson-Cook constitutive model of structural steel was modified on the basis of adiabatic warming effect and Perzyna constitutive model. Based on the result of the experiment, the fitting curves were drawn and compared with the revised constitutive model. The results showed that Q345 steel has obvious strain rate hardening effect and temperature soften effect, and the curves of the modified constitutive model and the experimental results matched very well. The modified Johnson-Cook constitutive relationship can precisely describe the dynamic characteristic of Q345 steel under the impact of temperature effect and strain rate effect.

\section{Introduction}

Fire and explosion can cause devastating personal injury and property damage. It has become one of major disasters which are harmful to human life and production. These explosions are hard to predict and very destructive. Whether the current frequent incidents of terrorism, or our lives in the accident, study on the anti explosion performance of structure is gradually changed to the development trend of future research. The constitutive relation of metal material has high strain rate dependent and high temperature dependent characteristics. The key is to accurately reflect the sensitivity of materials and to establish a simple and practical model ${ }^{[1]}$. Therefore, the test and application of elevated temperatures and high strain rate constitutive relation of steel has become a hot research topic in the present research.

The Johnson-Cook model ${ }^{[2]}$ is simple and the clear physical meaning. The parameters in the model can be directly determined by the SHPB test, so it has been widely used in the steel constitutive relation study. This test in the paper adopts the SHPB testing technique, and the impact experiment under different stain rates and different temperatures for Q345 steel which is widely used in steel structures are carried out. Considering high temperature effect caused by high speed deformation softening on stress strain curve, through fitting the test curve and modifying the Johnson-Cook model, the developed model can well reflect the combination of high temperature thermal effect and high strain rate effect of structural steel.

\section{Test of Material Properties}

Equipment and Principles of Test. Experimental studies on the high-temperature material properties of Q345 steel which is widely used in steel structures are carried out in the paper. The dynamic impact compression test was conducted by SHPB apparatus combined with pulse shaping 
techniques to study Q345 steel under high temperature and high strain rate conditions. The material of the impact bar, the incident bar and the transmission bar are $18 \mathrm{Ni}$ maraging steel. Basic parameters of experimental equipment: For the impact bar, the length is $300 \mathrm{~mm}$, the diameter is $14 \mathrm{~mm}$, the density is $7800 \mathrm{~kg} / \mathrm{m}^{3}$; For the incident bar, the length is $1200 \mathrm{~mm}$, the diameter is $14 \mathrm{~mm}$, the density is $7800 \mathrm{~kg} / \mathrm{m}^{3}$; For the transmission bar, the length is $1200 \mathrm{~mm}$, the diameter is $14 \mathrm{~mm}$, the density is $7800 \mathrm{~kg} / \mathrm{m}^{3}$. The experimental apparatus is shown in Fig. 1 .

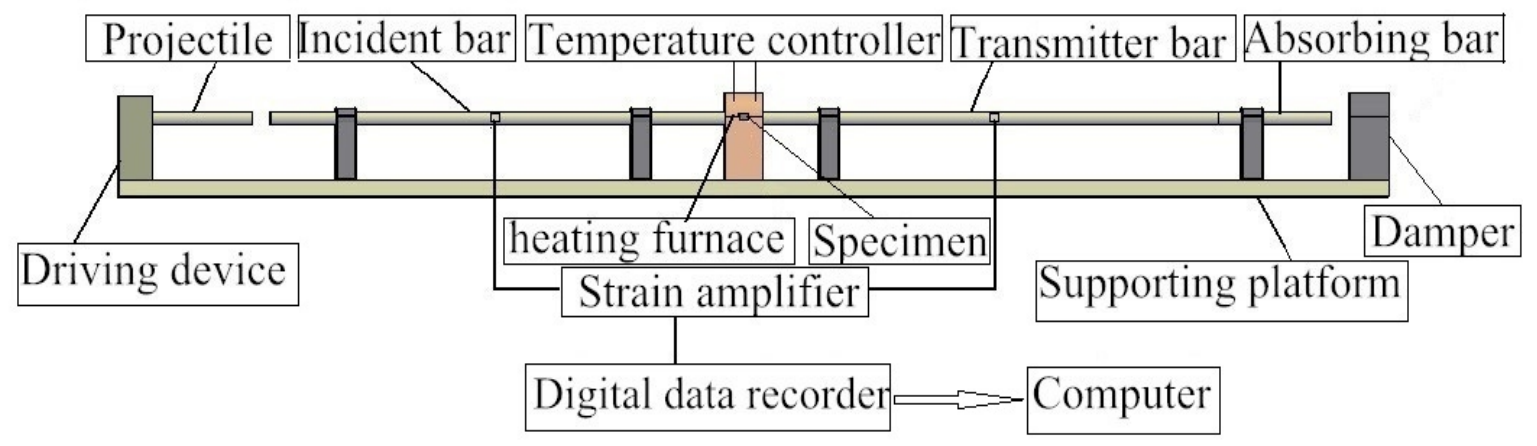

Fig. 1. The schematic of the Split Hopkinson Pressure Bar test

The experimental principle: in the heating furnace, the heating control system can keep the temperature of the test piece in the setting temperature when the test piece reaches the preset temperature; when the driving device is started, the air pressure in the driving device pushes the impact bar to strike the incident bar, and an incident wave is generated in the incident bar; the incident wave is transmitted to the specimen through the incident bar, so that the specimen is deformed; the incident wave is divided into two parts, and one part of the incident wave is reflected back, and a reflection wave is generated in the incident bar; the other part of the incident wave is passed into the transmitter bar, and a transmission wave is generated in the transmitter bar; these waves through the strain amplifier transfer to the super dynamic strain instrument, and the transient waveform storage into the computer. Formula for processing data:

$$
\begin{aligned}
& \varepsilon(t)=-\frac{2 C_{0}}{L} \varepsilon_{R}(t) \\
& \varepsilon(t)=-\frac{2 C_{0}}{L} \int \varepsilon_{R}(t) d t \\
& \sigma=E \frac{A}{A_{0}} \varepsilon_{T}(t)
\end{aligned}
$$

In the Formula: $\varepsilon(t)$ is strain rate; $\varepsilon(t)$ is strain; $\sigma$ is flow stress; $\mathrm{C}_{0}$ is the velocity of elastic wave propagation in an elastic bar; $L$ is length of specimen after deformation; $A$ is the cross section area of elastic bar; $A_{0}$ is the cross section area of specimen; $E$ is the young modulus of elastic bar; $\varepsilon_{R}$ and $\varepsilon_{T}$ are the reflection stress pulse and the transmission stress pulse, respectively.

Experimental result analysis. Q345 steel is used in this experiment. The size of the test piece is $\phi 4 \mathrm{~mm} \times 4 \mathrm{~mm}$. The test results are shown in Fig. 2. It can be seen from the figure that the obvious difference of yield strength is observed in the stress-strain curves of Q345 steel in different temperature. It shows that Q345 steel has a high temperature softening effect. When the strain rate is $3200 \mathrm{~s}^{-1}$, the yield strength curve is higher than the yield strength curve when the strain rate is $2300 \mathrm{~s}^{-1}$. It shows that Q345 steel has obvious strain rate strengthening effect. 


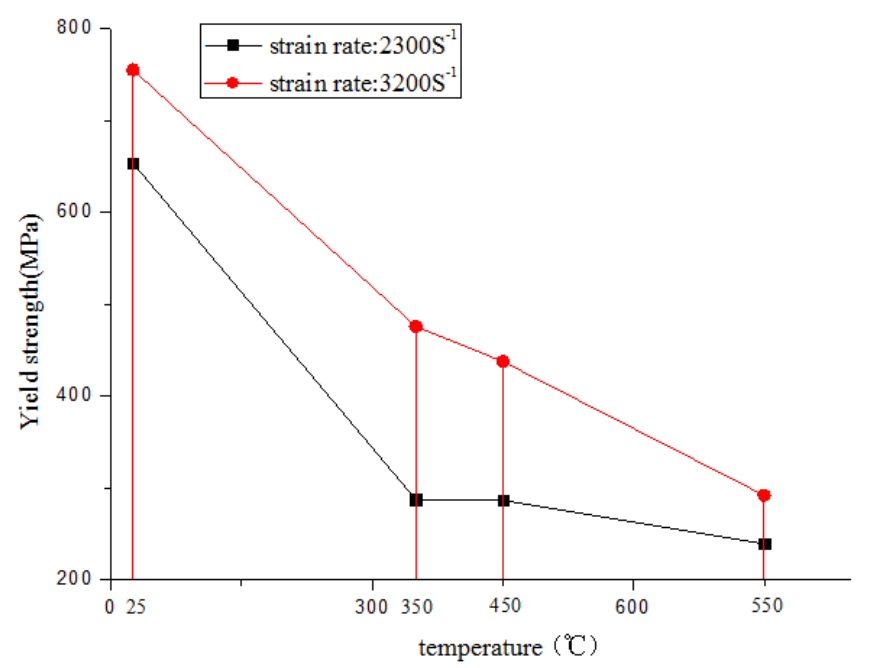

Fig. 2 yield strength of Q345 steel at different strain rates and different temperatures

\section{Constitutive relation}

Johnson-Cook model. The Johnson-Cook model is a mechanical model proposed by Johnson and Cook in 1983, and an improvement to the Ludwik equation which can reflect the dynamic characteristics of metal $^{[3]}$. Johnson-Cook model is generally used to describe the constitutive relation of metallic materials in the case of large deformation, high strain rate and high temperature, to reflect the strain rate hardening effect and high temperature softening effect of steel. The function relation of stress and strain, strain rate and temperature can be presented well. Its model can be expressed as:

$$
\sigma_{y}=\left(A+B \varepsilon_{l}^{n}\right)\left[1+C \ln \frac{\& \&}{8 \delta}\right]\left[1-\left(T^{*}\right)\right]
$$

In the Formula: $\mathrm{A}, \mathrm{B}, \mathrm{C}, \mathrm{n}$ and $\mathrm{m}$ five parameters are determined by experiment; $\sigma_{y}$ is equivalent stress of steel considering strain rate and temperature; $\varepsilon_{l}$ is equivalent plastic strain; \& \& is reference strain rate; $\& \delta$ is the strain rate should be considered in the calculation; $T^{*}$ is dimensionless temperature, $T^{*}=\left(T-T_{r}\right) /\left(T_{m}-T_{r}\right), T$ is test temperature; $T_{r}$ is room temperature; $T_{m}$ is melting temperature of material. Johnson-Cook constitutive model is established for better numerical simulation, but the constitutive equations have not fully considered the thermal history effect and the strain rate history effect. It simply multiplies the parameters of strain, strain rate, temperature that affect yield strength, in order to determine the undetermined coefficients in the model in experiment ${ }^{[4]}$.

Modification of Constitutive Equation. As above-mentioned, there are many defects in the establishment of the Johnson-Cook model. Because the model does not consider the viscous properties of the metal, the Perzyna model is introduced in this paper ${ }^{[5]}$ :

$$
\sigma_{y}^{\prime}=\left[1+\left(\frac{\varepsilon^{p l}}{\gamma}\right)^{m}\right] \sigma_{y}
$$

In the Formula: $\sigma_{y}^{\prime}$ is yield stress considering strain rate effect; $\varepsilon^{p l}$ is equivalent plastic strain rate; $m$ is strain rate enhancement factor; $\gamma$ is viscosity coefficient; $\sigma_{y}$ is yield stress. 
The stress model of Perzyna did not reflect the temperature dependent yield strength, so combining the advantages and disadvantages of these two equations, the $\mathrm{J}-\mathrm{C}$ model is modified to:

$$
\sigma_{y}=\left(\sigma_{0}+B \frac{\varepsilon^{N}}{C^{N}+\varepsilon^{N}}\right)\left[1+\ln \frac{\varepsilon \&}{\varepsilon_{0}}\right]\left[1-(T(t))^{m}\right]
$$

One of the main differences between the structural analysis at room temperature and the structural analysis under high temperature is that the material properties of the steel should be considered with the change of temperature. The greatest impact is the change of the mechanical properties of the material at high temperature ${ }^{[6]}$. The impact load causes the material to produce the plastic deformation, and the plastic work which is produced by the impact is changed into heat energy, and some of the heat is dissipated into the surrounding environment and a part of the heat is transferred into the material which leads to the increase of the temperature of the metal. As above-mentioned, the large strain rate plastic work turns into heat energy which can be used to calculate the temperature of the material $^{[7]}$ :

$$
\Delta T(\varepsilon)=\frac{\eta}{\rho C_{v}} \int_{0}^{\varepsilon} \sigma d \varepsilon
$$

So, integrated by the improved Johnson-Cook model and the above temperature calculation formula, the coefficient $\mathrm{K}$ and strain rate dependent terms was added in third temperature softening items $^{[8]}$. The improved constitutive model:

$$
\begin{aligned}
& \sigma_{y}=\left(\sigma_{0}+B \frac{\varepsilon^{N}}{C^{N}+\varepsilon^{N}}\right)\left[1+\ln \frac{\varepsilon \&}{\varepsilon_{0}}\right]\left[1-\left(\frac{T+\left\langle\frac{8 \&-1}{|\& \&-1|}\right\rangle k-T_{R}}{T_{m}-T_{r}}\right)^{m}\right] \\
& \left\langle\frac{8 \&-1}{|\& \&-1|}\right\rangle=\left\{\begin{array}{cl}
1 & \dot{\varepsilon}> \\
0 & \dot{\varepsilon}<
\end{array}\right.
\end{aligned}
$$

Fitting of constitutive equation and test data. Comparison the fitting curves between the initial and modified J-C model, as shown in Fig. 3, it is concluded that the modified constitutive relation can be better used in the study of the dynamic response of steel under high temperature and high strain rate.
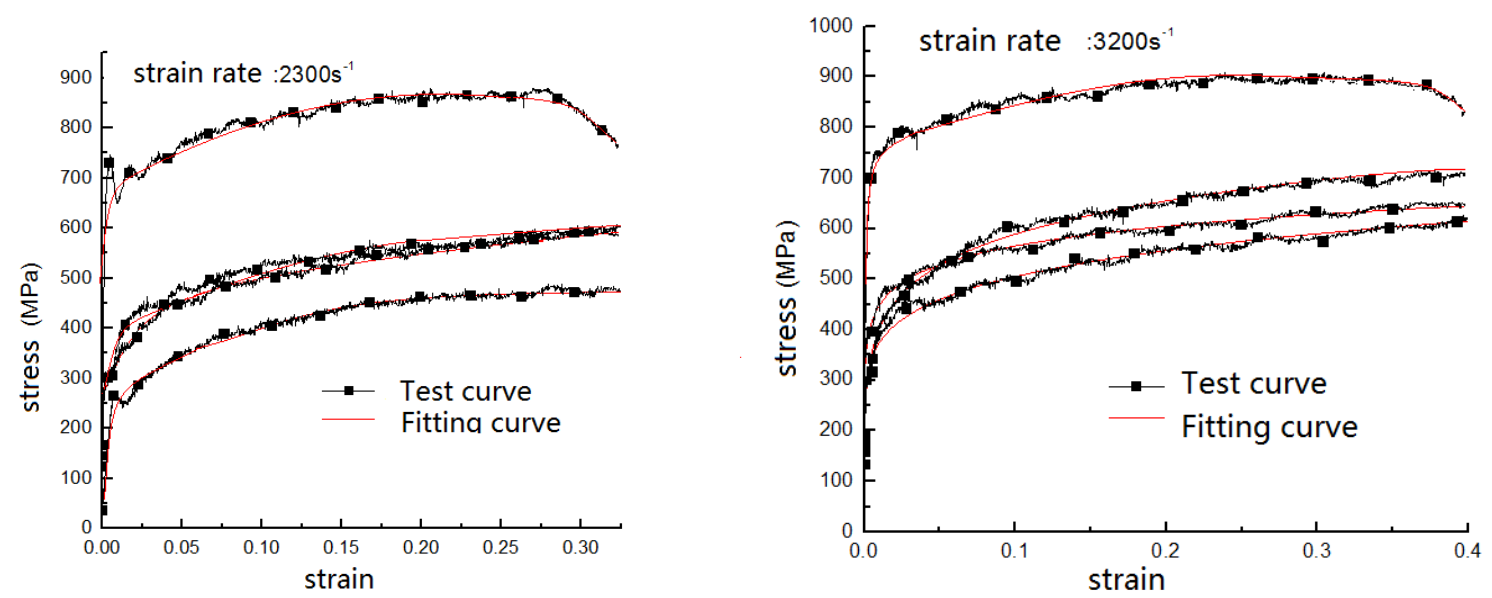

Fig. 3. Comparison between the fitting results of the modified Johnson-Cook model and the experimental results 


\section{Conclusions}

Through the compare and combination of the advantages of Perzyna model and Johnson-Cook model, and the temperature rise caused by the change of the plastic work in the adiabatic process, the Johnson-Cook model is modified.

By fitting the experimental results and the modified Johnson-Cook model, the constitutive model parameters of Q345 steel under different strain rates and different temperatures were obtained, and through the comparison of the test curve and fitting curve confirmed that the model can better describe quality carbon steel effect of temperature and strain rate effect.

\section{Acknowledgements}

This work was financially supported by the Liaoning Natural Science Foundation of China (20141075), Open Foundation of Key State Laboratory of Explosive Science and Technology of China (KFJJ10-14M), Open Foundation of Jiangsu Key Laboratory of Environmental Impact and Structural Safety in Engineering of China (JSKL2014K10), Liaoning Province Education Department Foundation of China (L2015448) and the Department of Housing and Urban-rural Development of Science and Technology Plan of China (2015-K2-018).

\section{References}

[1] S. F. Chen and Q. Gu: Steel Structure (China Building Industry Press, China 2007). (in Chinese)

[2] C. R. Johson and W. H. Cook in: Proceedings of the 7th International Symposium on Ballistics, The Hague, 19-21 April 1983, pp. 541-547.

[3] M. A. Bradford, T. K. Luu, A. Heidarpour. Journal of Constructional Steel Research Vol. 64 (2008), p. 732.

[4] S. Yan, Z. Q. Xin, et al. Journal of Shenyang Jianzhu University (Natural Science) Vol. 30 (2014), p. 593.

[5] P. Perzyna. Quarterly of Applied Mathematics Vol. 20 (1963), p. 321.

[6] G. Q. Li, K. Chen, S. C. Jiang et al. Building Structure Vol. 31(2001), p. 53. (in Chinese)

[7] S. Nemat-Nasser and W. G. Guo. Mechanics of Materials Vol. 35 (2003), p. 1023.

[8] B. Shang, Jing Sheng, B. Z. Wang. Explosion and Shock Waves Vol. 28 (2008), p. 527. 